\title{
Chronisches Nierenversagen
}

\author{
Update 2015
}

Christoph Wanner, Markus Ketteler

\section{Was ist neu?}

- Studien zur Progressionsverzögerung diabetischer Spätschäden: Ergebnisse aus großen Studien mit antidiabetischer Medikation zu mikrovaskulären Endpunkten (Nierenfunktion) werden noch 2015 publiziert und zur Verfügung stehen.

- Kardiovaskuläre Protektion bei Niereninsuffizienz: Weniger als die Hälfte der Patienten mit chronischen Nierenerkrankungen erhalten eine cholesterinsenkende Therapie. Die Umsetzung von Leitlinien bedarf der Ausweitung der Statintherapie.

- Progressionsverzögerung bei diabetischer Nephropathie: Die Inzidenz chronischer Dialysebehandlung ist europaweit rückläufig und könnte auf die verbesserte Prävention zurückgeführt werden.

- Seltene Nierenerkrankungen: Seltene, genetisch bedingte Nierenerkrankungen werden zunehmend entschlüsselt und Therapien stehen zur Verfügung (Enzymersatztherapien, Chaperone, small molecules, Substrate, Vasopressin Antagonist).

\section{Studienprogramme zur Progressionsverzögerung diabetischer Spätschäden}

Gegenwärtig befinden sich mehr als 200000 Typ2-Diabetiker in Zulassungsstudien neuer Substanzen und wissenschaftlichen Untersuchungen. Derzeit werden die Sicherheit und Wirksamkeit von DPP-4-Inhibitoren [1], SGLT2-Inhibitoren, einem Endothelin-A-Rezeptor-Antagonisten (Atrasentan) und einem Mineralkortikoidrezeptor-Antagonisten (Finerenon) in klinischen Outcomestudien geprüft. Viele weitere Strategien befinden sich in Phase-2b-Studien.

Je nach Wirkung der Interventionen sind die Endpunkte kardiovaskulär oder renal:

- 3 Punkt MACE (major adverse cardiac event)

- Verdoppelung des Serumkreatinins

- $40 \%$ Rückgang der geschätzten glomerulären Filtrationsrate (eGFR)

- Dialysepflichtigkeit.

Die Albuminurie, ein Surrogatendpunkt, ist meist in den sekundären Endpunkten verankert. Albuminurie wird in 3 Stadien unterteilt:

- Stadium 1: normale Albuminausscheidung

- Stadium 2: Mikroalbuminurie

- Stadium 3: Makroalbuminurie

\section{Klinische Relevanz}

Derzeit werden große Anstrengungen (Studienprogramme) unternommen, um die Komorbiditäten des Typ-2-Diabetikers einzudämmen und die Progression der diabetischen Nephropathie zu verzögern oder sogar zu stabilisieren.

\section{Kardiovaskuläre Protektion bei Niereninsuffizienz}

Die wenigsten Patienten mit chronischer Nierenerkrankung und/oder diabetischer Nephropathie erreichen das Stadium der terminalen Niereninsuffizienz. Viele Patienten sterben während der Progression der Nierenerkrankung an kardiovaskulären Komplikationen [2]. Ziel ist es, beide Endstrecken - die kardiovaskuläre Komplikation und gleichzeitig die Dialysepflichtigkeit - durch Prävention zu verzögern oder zu vermeiden. Kardiovaskuläre Basisprävention bedeutet gleichzeitig, die Progression der chronischer Nierenerkrankung zu verzögern. Sie orientiert sich am bestehenden Risiko des Patienten und besteht aus - Blutdrucksenkung, die einen RAS-Blocker beinhalten sollte, und

- aus der Senkung des LDL-Cholesterin durch ein Statin.

Für letzteren therapeutischen Ansatz hatte die SHARP-Studie unzweifelhaft nachgewiesen, dass eine LDL-Senkung die kardiovaskuläre Ereignisrate bei Patienten mit chronischer Nierenerkrankung signifikant senkt [3].

Basisprävention bei Patienten mit Typ-2-Diabetes und diabetischer Nephropathie sollte eine Aufgabe in der breit angelegten Basisversorgung sein und bedarf nicht notwendigerweise eines Nephrologen. Spezialmedizin kann zusätzlich über die Vermeidung des sekundären Hyperparathyreoidismus, der renalen Anämie und einer Azidose angeboten werden.

Bei den mehr als 5300 Patienten mit chronischem Nierenversagen (770 davon mit diabetischer Nephropathie [Höchstrisikogruppe]) in der deutschen Chronic-Kidney-Disease-Kohorte (GCKD) waren nur ca. $50 \%$ mit einem Statin versorgt [4]. Entsprechend den KDIGO-Leitlinien (Kidney Disease Improving Global Outcome) sollte den meisten Patienten mit chronischem Nierenversagen mit einer eGFR $<60 \mathrm{ml} / \mathrm{min} / 1,73 \mathrm{~m}^{2}$ eine 
Statintherapie in moderater Theapieintensität angeboten werden (Evidenzlage 1A) [5].

Warum die Hälfte aller Patienten mit chronischer Nierenerkrankung und/oder diabetischer Nephropathie ein bewährtes Therapieprinzip nicht erhält, ist nicht bekannt und bedarf der weiteren Untersuchung (z.B. Follow-up-Beobachtungen der GCKD-Patienten). Erklärt wird dies mit folgende Hypothesen:

- Nephrologen fokussieren derzeit bevorzugt auf den Blutdruck.

- Patienten sind meist bereits mit einer häufig notwendigen 4-fach antihypertensiven Therapie überfordert. Jede weitere Komedikation senkt die Medikamentenadhärenz.

- Die Ergebnisse der 2011 publizierten SHARPStudie [3] sind noch nicht ausreichend bekannt.

- Der LDL-Cholesterinsenkung wird zu wenig Wert beigemessen.

- Myopathie-Nebenwirkungen sind bei Einsatz in der Breite ein limitierender Faktor bei Nierenkranken.

Allerdings hätte letzteres Argument gerade bei dem großen Kollektiv der SHARP-Studie (>9000 Patienten) sichtbar werden müssen. Letztendlich sollten aber alle Spielräume bei der konservativen Basisprävention genutzt werden, bevor man sich anderen wenig gesicherten Therapieformen zuwendet.

\section{Klinische Relevanz \\ Die Implementierung von Leitlinien zur Prävention kardiovaskulärer Komplikationen verlangt eine deutlich Ausweitung der Verschreibungsrate von Statinen und kardiovaskulär präventiver Medikation bei Nierenkranken.}

\section{Progressionverzögerung bei diabetischer Nephropathie}

Vor einem Jahr waren wir an dieser Stelle noch skeptisch, ob in naher Zukunft Verbesserungen in der Therapie der diabetischen Nephropathie erzielt werden können [6]. Diese Skepsis bleibt weiterhin erhalten, ist aber durch die Beurteilung der gegenwärtigen Situation etwas geändert worden. Folgende Beobachtungen wurden in diesem Jahr mitgeteilt:

- Die Zahl der neu in ein Dialyseprogramm aufgenommenen Patienten mit Typ-2-Diabetes (Inzidenz) nimmt nicht mehr zu, sondern ist europaweit rückläufig (minus 2,2\%) (Vortrag M. Pippias - ERA-EDTA Kongress London 2015 und [7]). Im Österreichischen Register nimmt die Zahl der Typ-2-Diabetiker im Dialyseprogramm (Prävalenz) sogar schon ab.

- Die Zahl der Patienten mit Typ-2-Diabetes, die eine Makroalbuminurie aufweisen, geht deutlich zurück, und hohe Albuminmengen im Urin sind eine Rarität geworden (Querschnittsbeobachtungen; persönliche Mitteilung)

- In den Studien BEACON [8] und ALTITUDE [9] befanden sich bis zu 20\% Normoalbuminuriker unten den Patienten mit fortgeschrittener diabetischer Nephropathie und der Nierenfunktionsverlust lag bei nur $2-4 \mathrm{ml} / \mathrm{min} / 1,73 \mathrm{~m}^{2}$ eGFR pro Jahr.

Es besteht breiter Konsens, dass bei Patienten mit Typ-2-Diabetes und gut eingestelltem Blutdruck sowie geringer Albuminurie die Nierenfunktion über viele Jahre stabilisiert werden kann. Begleitend hilfreich ist eine Natriumrestriktion (entsprechend $130 \mathrm{mmol} / 24 \mathrm{~h}$-Urin) und eine entsprechend angepasste Ernährung. Den Rückgang der Dialysepflichtigkeit assoziieren wir mit einer erfolgreichen Implementierung dieser Interventionen. Allerdings kann dieser Status Quo auch nur ein vorübergehender Erfolg sein, denn die Bevölkerung altert derzeit deutlich, und ohne gesundes Altern wird die Inzidenz für Dialyse bei Typ-2Diabetes in absehbarer Zeit wieder ansteigen.

Darüber hinaus wurden diese Daten im europäischen Ausland erhoben und müssen auf Deutschland nicht zwingend zutreffen. Deutschland hat kein Dialyse- oder Prädialyseregister. Mit dem Ende des Registers QUASI-Niere im Jahr 2006 können wir in Deutschland nur annehmen, dass die Entwicklungen bei uns so ähnlich stattfinden wie z.B. im Nachbarland Österreich.

\section{Klinische Relevanz}

Die Zahlen an Patienten, die eine Dialysebehandlung bedürfen (Inzidenz) sind europaweit und in den USA rückläufig. Es wird vermutet, dass Präventionsmaßnahmen, wie eine verbesserte Blutdruckeinstellung, dafür verantwortlich sein könnten.

\section{Seltene Nierenerkrankungen}

Etwa $80 \%$ der ca. 7000 bekannten seltenen Erkrankungen haben eine genetische Ursache. Darunter befinden sich etwa 200 monogene Nierenerkrankungen. D. h. einer von 5 Patienten mit Niereninsuffizienz leidet neben den vaskulären und diabetischen Nierenerkrankungen an einer seltenen Erkrankung. Meist können hier weitere erst- bzw. zweitgradig verwandte Familienmitglieder mit Niereninsuffizienz identifiziert werden [10]. Bisher wurde viel zu wenig auf das unmittelbare Patientenumfeld fokussiert und Stammbäume werden nur selten gezeichnet. Mit der Einführung von vielfältigen OrphanDrug-Therapien werden diese Erkrankungen und deren therapeutisches Potenzial sichtbarer. Wichtig ist es deshalb, potenziell genetische Krankheitsgruppen zu erkennen (zystische Nierenerkrankungen, Fabry-Nephropathie, Lateonset-Zystinose, Erkrankungen des Tubulussys- 


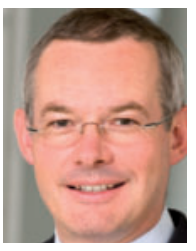

Prof. Dr. Christoph Wanner ist Oberarzt der Medizinischen Klinik und Poliklinik I und Leiter der Abteilung Nephrologie am Universitätsklinikum Würzburg. wanner_c@ukw.de

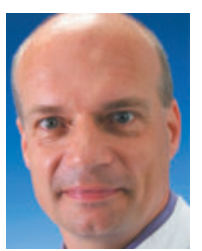

Prof. Dr. Markus Ketteler ist Chefarzt der Nephrologischen Abteilung der Medizinischen Klinik III am Klinikum Coburg markus.ketteler@klinikumcoburg.de tems, Ziliopathien) und dann den jeweils spezifischen Therapien zuzuführen (z. B. Enzymersatz).

In Kürze wird Tolvaptan, ein ADH-Antagonist, den Patienten mit polyzystischer Nierendegeneration (ADPKD) in den Niereninsuffizienz-Stadien 1-3 zur Verfügung stehen. Die Zulassung dieser kostenträchtigen Therapie wird einige Einschränkungen beinhalten, um vor allem die Patienten vor den Nebenwirkungen der Behandlung (u.U. erhebliche Polyurie) besser zu schützen.

Es ist durchaus sinnvoll, bei seltenen Erkrankungen mit Referenzzentren zusammenzuarbeiten, um auch den weiteren Erkenntnisgewinn durch Registerpflege der seltenen Erkrankungen weiterzuführen.

Die überwiegende Mehrheit aller Kinder und Jugendlichen, die dialysepflichtig werden, leiden an einer seltenen genetischen Erkrankung. Aber nicht alle Kinder mit kongenitalen Anomalien der Nieren und der ableitenden Harnwege (CAKUT) werden im Kindesalter dialysepflichtig. Über die Hälfte erreicht das Erwachsenenalter [11] mit Eintritt der terminalen Niereninsuffizienz zwischen dem 20. und 50. Lebensjahr. Sogenannte „second-hit" Insulte und Risikofaktoren sind von Bedeutung, wie beispielsweise Übergewicht und hoher Blutdruck. Die Transitionsphase vom Adoleszenten in das Erwachsenenalter stellt eine Herausforderung für die kontinuierliche Betreuung Nierenkranker dar.

Die Überlegungen und Zahlen weisen auf eine Unterdiagnose angeborener Erkrankungen hin, die sich in der Diagnose chronische Glomerulonephritis oder im Segment Schrumpfnieren und „unbekannt“ verbergen.

\section{Klinische Relevanz}

- Die diabetische und die vaskuläre Nephropathie sind die Hauptursachen für terminale Niereninsuffizienz.

- Darüber hinaus sind wahrscheinlich ein Viertel der erwachsenen mit chronischer Niereninsuffizienz von einer genetischen Grunderkrankung betroffen.

- Die Stammbäume der Patienten mit seltenen genetisch bedingten Nierenerkrankungen sollten beachtet werden.

- Neue Therapien für seltene genetische Erkrankungen stehen in immer größeren Umfang zur Verfügung.

\section{Literatur}

1 Green HB, Ethel MA, Armstrong PW et al. Effect of sitagliptin on cardiovascular outcomes in type 2 diabetes. N Engl J Med 2015; 373: 232-242

2 Navaneethan SD, Schold JD, Arrigain S et al. Cause-specific death in non-dialysis-dependent CKD. J Am Soc Nephrol 2015; DOI 10.1681/ASN.2014101034

3 Baigent C, Landray M], Reith C et al. The effects of lowering LDL cholesterol with simvastatin plus ezetimibe in patients with chronic kidney disease (Study of Heart and Renal Protection): a randomised placebo-controlled trial. Lancet 2011; 377: 2181-2192

4 Schneider MP, Hübner J, Titze S et al. Implementation of the KDIGO Guideline on Lipid Management would require substantial increase in statin prescription rates: an analysis from the GCKD cohort. Kidney Int 2015; in press

5 Wanner C, Tonelli M. Lipid management in chronic kidney disease: synopsis of the KDIGO 2013 clinical practice guideline. Kidney Int 2014; 85: 1303-1309

6 Wanner C, Ketteler M. Chronisches Nierenversagen Update 2014. Dtsch Med Wochenschr 2014; 139 : 261-263

7 Kramer A, Stel SV, Abad Diez JM et al. Renal replacement therapy in Europe - a summary of the 2010 ERA-EDTA Registry Annual Report. Clin Kidney J 2013; 6: 105-115

8 Parving $\mathrm{HH}$, Brenner BM, McMurray || et al. ALTITUDE Investigators. Cardiorenal end points in a trial of aliskiren for type 2 diabetes. N Engl ] Med 2012; 367: 2204-2213.

9 De Zeeuw D, Akizawa T, Audhya P et al. Bardoxolone Methyl in Type 2 Diabetes and Stage 4 Chronic Kidney Disease. N Engl J Med. 2013; 369: 2492-2503

10 Devuyst O, Knoers NV, Remuzzi G et al. Rare inherited kidney diseases: challenges, opportunities, and perspectives. Lancet 2014; 383: 1844-1859.

11 Wühl E, van Stralen KJ, Verrina E et al. Timing and outcome of renal replacement therapy in patients with congenital malformations of the kidney and urinary tract. Clin J Am Soc Nephrol 2013; 8: $67-74$
Interessenkonflikt

Die Autoren erklären, dass kein Interessenkonflikt besteht.

DOI 10.1055/s-0041-103753

Dtsch Med Wochenschr 2015; 140: 1216-1218

(c) Georg Thieme Verlag KG . Stuttgart · New York .

ISSN 0012-0472 